\title{
A Helmholtz-Lie Type Characterization of Ellipsoids, I
}

\author{
P. M. Gruber \\ Abteilung für Analysis, Technische Universitaet Wien, \\ Wiedner Hauptstraße 8-10/1142, A-1040 Vienna, Austria \\ pmgruber@pop.tuwien.ac.at
}

\begin{abstract}
A closed convex surface $S$ in $\mathbb{E}^{d}$ with $d$ odd, is an ellipsoid if and only if it has the following property: for any pair of points $x, y$ in $S$ there is an affine transformation which maps $x$ onto $y$ and a suitable neighborhood of $x$ in $S$ onto a neighborhood of $y$ in $S$.
\end{abstract}

\section{Introduction and Statement of Result}

1.1. Results of Helmholtz-Lie type are characterizations of special quadrics or classical spaces and geometries by the property that there are local or global self-mappings of the surface or space which preserve the local geometric structure. In general "many" such affinities, isometries, or homeomorphisms are required. A survey of the literature up to 1956 is due to Freudenthal [10]. It covers in particular the contributions of Helmholtz, Lie, Weyl, Kolmogorov, Birkhoff, and Tits.

To convey the spirit of such results we cite a theorem of Laugwitz [15] which he proved in the context of affine differential geometry. Let $S$ be a hypersurface of class $\mathscr{C}^{3}$ in Euclidean $d$-space $\mathbb{E}^{d}$ with the following property: for any $x, y \in S$ and tangent directions $s, t$ of $S$ at $x$ and $y$, respectively, there is a unique linear transformation of $\mathbb{E}^{d}$ which maps $x$ onto $y$, a suitable neighborhood of $x$ in $S$ onto a neighborhood of $y$ in $S$ and, finally, $s$ onto $t$. Then $S$ is a special quadric.

1.2. If instead of general hypersurfaces, closed convex surfaces are considered it seems plausible that the conclusion of the above result of Laugwitz or of its predecessors due to Helmholtz, Lie, and Weyl holds but with less assumptions. Before stating our result we review some pertinent results from convex geometry.

Let $S$ be a closed convex surface in $\mathbb{E}^{d}$, that is the boundary of a compact convex set in $\mathbb{E}^{d}$ with nonempty interior. If the group of projectivities which map $S$ onto 
itself is transitive on $S$, then $S$ is an ellipsoid. This result of Auerbach [2] and Busemann [8] generalizes the following classical characterization of ellipsoids of Brunn [5]: if $S$ admits affine reflections in all directions, then it is an ellipsoid. For a different generalization of Brunn's result see [12].

Assume now that $S$ is of class $\mathscr{C}^{3}$ and with positive Gaussian curvature. $S$ is an ellipsoid if for any $x, y \in S$ there is a volume-preserving affinity which maps $x$ onto $y$ and a suitable neighborhood of $x$ in $S$ onto a neighborhood of $y$ in $S$. This is a consequence of a result of Blaschke [4, p. 35], saying that $S$ is an ellipsoid if its affine curvature is constant and positive. A more refined result of this type was given in a preliminary version of an article by Berger [3] in the context of the problem of the existence of caustics of convex surfaces.

1.3. The aim of this article is to prove the following convexity result.

Theorem. Let $S$ be a closed convex surface in $\mathbb{E}^{d}, d$ odd, with the following property: for any pair of points $x, y$ in $S$ there is an affine transformation of $\mathbb{E}^{d}$ which maps $x$ onto $y$ and a suitable neighborhood of $x$ in $S$ onto a neighborhood of $y$ in $S$. Then $S$ is an ellipsoid.

Our proof makes use of the differentiability results of Alexandrov for convex surfaces, of a characterization of ellipsoids due to Burton by the property that their planar sections are centrally symmetric, and of a topological result of Mani on continuous fields of congruent planar convex curves tangent to the unit sphere $S^{d-1}$. In addition, Baire's category theorem and a result on invariant subspaces of symmetric matrices are used. Unfortunately Mani's result holds only for odd $d$. While this prevents the extension of our proof to all dimensions, we conjecture that the theorem holds for all $d$.

\section{Tools and Preliminaries}

In the following when we speak of a convex curve, a circle, or an ellipse, we actually mean a convex surface, a sphere, or an ellipsoid in $(d-1)$-dimensional space. By area the $(d-1)$-dimensional measure is meant. A general reference on convexity is [20].

Let $S$ be a closed convex surface in $\mathbb{E}^{d}$ which is smooth and strictly convex, that is, it is of class $\mathscr{E}^{1}$ and contains no line segment.

2.1. For $x \in S$ let $H(x)$ be the supporting hyperplane and let $n(x)$ be the interior normal unit vector of $S$ at $x$. For $x \in S$ and $\delta>0$ we consider the convex curve

$$
S(x, \delta)=(H(x)+\delta n(x)) \cap S .
$$

If

$$
\frac{1}{\sqrt{2 \delta}}(S(x, \delta)-(x+\delta n(x)))
$$


tends to a limit as $\delta \rightarrow+0$ in the sense of the closed Hausdorff limit, the limit is called the indicatrix $I(x)$ of $S$ at $x$. The following proposition is well known.

(1) Let $x \in S$ then, if $I(x)$ is bounded,

$$
\frac{1}{\sqrt{2 \delta}}(S(x, \delta)-(x+\delta n(x))) \stackrel{\delta^{\mathrm{H}}}{\longrightarrow} I(x)
$$

Here $\delta^{\mathrm{H}}$ is the Hausdorf metric; that is, for nonempty compact subsets $C, D$ of $\mathbb{E}^{d}$ their Hausdorff distance $\delta^{\mathrm{H}}(C, D)$ is the maximum distance which a point of one of the sets $C, D$ can have from the other set.

A result of Alexandrov [1] says that in the sense of the ordinary surface area measure on $S$,

(2) the indicatrix $I(x)$ exists and is a quadric at almost all $x \in S$.

See [19].

2.2. An immediate consequence of a result of Burton [6] is the following characterization of ellipsoids:

(3) Assume that for each $x \in S$ the convex curve $S(x, \delta)$ is centrally symmetric for all sufficiently small $\delta>0$. Then $S$ is an ellipsoid.

2.3. A topological space is Baire if any countable union of nowhere dense subsets has dense complement. By Baire's category theorem a complete metric space is Baire. Let $S$ be endowed with the metric and topology induced by that of $\mathbb{E}^{d}$. Since $S$ is a closed subset of $\mathbb{E}^{d}$, it is complete metric. Hence,

(4) if $S$ is a countable union of closed subsets, then at least one of these has nonempty interior in $S$.

2.4. A continuous field of affine-equivalent convex curves tangent to $S^{d-1}$ is a family of affine-equivalent convex curves $S(u): u \in S^{d-1}$ with the following properties:

(i) Each $S(u)$ is contained in the supporting hyperplane of $S^{d-1}$ at $u$.

(ii) $u$ is the center of the (unique) ellipse $E(u)$ of minimum area containing $S(u)$.

(iii) The mapping $u \rightarrow S(u)$ is continuous with respect to $\delta^{\mathrm{H}}$.

For properties of $E(u)$ see [9]. Note that $E(u)$ depends continuously on $S(u)$.

If in the above definition $E(u)$ is replaced by the circle $C(u)$ of minimum radius containing $S(u)$ and affine-equivalence by congruence, we speak of a continuous field of congruent convex curves tangent to $S^{d-1}$ instead.

Using topological tools, Mani [17] proved the following result:

(5) Let $d$ be odd. Then any continuous field of congruent convex curves tangent to $S^{d-1}$ consists of circles. 
In our proof of the theorem we need a slightly different version of (5):

(6) Let $d$ be odd. Then any continuous field of affine-equivalent convex curves tangent to $S^{d-1}$ consists of ellipses.

Burton [7, p. 315] made a very short remark on the (natural) proof of (6). Since it does not seem to be trivial, we indicate how (6) may be deduced from (5). For each $u \in S^{d-1}$ let $E_{u}$ be the symmetric $d \times d$ matrix corresponding to the ellipsoid with "poles" $\pm u$ and "equator" $E(u)-u$. The first step is to prove that the mapping

$$
u \rightarrow E_{u}: u \in S^{d-1} \quad \text { is continuous. }
$$

Next consider the eigenvalues and the corresponding invariant subspaces of $E_{u}$, say

$$
1, \lambda_{1}<\lambda_{2}<\cdots<\lambda_{k} \text { and } I_{0}, I_{1}, \ldots, I_{k}
$$

and define a linear transformation $L_{u}$ of $\mathbb{E}^{d}$ by

$$
L_{u}(x)=x_{0}+\frac{1}{\lambda_{1}} x_{1}+\cdots+\frac{1}{\lambda_{k}} x_{k} \quad \text { for } \quad x=x_{0}+\cdots+x_{k} \in \mathbb{E}^{d}, \quad x_{i} \in I_{i} .
$$

Then we have to show that the mapping

$$
u \rightarrow L_{u}: u \in S^{d \cdot 1} \quad \text { is continuous. }
$$

This may be done using a result of Stewart [21] on the sensitivity of invariant subspaces of symmetric matrices; see also p. 413 of [11]. The final step is to prove that

$$
L_{u}(S(u)): u \in S^{d-1}
$$

is a continuous field of congruent convex curves tangent to $S^{d-1}$. Applying (5) we obtain (6), as required.

\section{Proof of the Theorem}

Clearly, if $S$ is an ellipsoid it satisfies the assumptions of the theorem.

Conversely, let $S$ be a closed convex surface in $\mathbb{E}^{d}$ for which the assumptions of the theorem hold. In particular, $d$ is odd.

3.1. We begin the proof by introducing some further notation. Note that $S$ is endowed with the metric induced by the Euclidean metric of $\mathbb{E}^{d}$. For $x \in S$ and $\varepsilon>0$ let $N(x, \varepsilon)$ be the (open) $\varepsilon$-neighborhood of $x$ (in $S$ ). For $x, y \in S$ let $A_{x y}, B_{x y}, \ldots$, denote affine transformations of $\mathbb{E}^{d}$ which map suitable neighborhoods of $x$ onto neighborhoods of $y$. If we write $A_{x y}(N(x, \varepsilon))$ it is to be understood, particularly, that $N(x, \varepsilon)$ is contained in the neighborhood of $x$ which by $A_{x y}$ is mapped onto a neighborhood of $y$. The absolute value of the determinant of the 
linear part of $A_{x y}$ is denoted $\left|A_{x y}\right|$. For $\delta>0$ let $C(x, \delta)$ be the cap of $S$ with center $x$ and height $\delta$, that is the part of $S$ between $H(x)$ and $H(x)+\delta n(x)$. Obviously, its boundary (in $S)$ is $S(x, \delta)$, see 2.1. Let $|C(x, \delta)|$ be the volume of the convex hull of $C(x, \delta)$. $|C(x, \delta)|$ is called the volume of $C(x, \delta)$. If $\mu=|C(x, \delta)|$ we also write $C(x, \mu)$ instead of $C(x, \delta)$; similarly, $S(x, \mu)$ stands for $S(x, \delta)$.

3.2. Next we prove some simple properties of the affinities $A_{x y}$ and of $S$, starting with the following:

(7) $\left|A_{x y}\right|>0$ for all $x, y \in S$.

Otherwise there are $x, y \in S$ with $\left|A_{x y}\right|=0$. Then a suitable neighborhood of $x$ is mapped by $A_{x y}$ onto a-planar-neighborhood of $y$. By the assumptions of the theorem this in turn implies that any point $z \in S$ has a planar neighborhood (consider the pair $y, z$ ). However, clearly, this is impossible if $z$ is a point where a circumsphere of $S$ touches $S$, concluding the proof of (7).

(8) $S$ is smooth and strictly convex.

To see this choose for $x$ first a point where an insphere of $S$ and then a point where a circumsphere of $S$ touches $S$ and apply (7) together with the assumptions of the theorem.

(9) $I(x)$ exists and is an ellipse for each $x \in S$.

By (2) there is a point in $S$ where the indicatrix exists and is a quadric. Then (7) and the assumptions of the theorem show that it exists and is a quadric at each point of $S$. Next, at a point where a circumsphere of $S$ touches $S$ the indicatrix of $S$ is contained in a circle; this is an immediate consequence of the definition in 2.1. Thus by (7) and our assumptions it is bounded at each point of $S$. A similar argument, but using an insphere, shows that at each point of $S$ the indicatrix contains a small circle. Taken together, these properties yield (9).

The remaining part of the proof of the theorem is split into two cases.

3.3. Case 1. Firstly, we assume that

(10) there are points $p, q \in S$ and affine transformations $A_{p q}, B_{p q}$ with $\left|A_{p q}\right| \neq$ $\left|B_{p q}\right|$.

3.3.1. By (7) both $A_{p q}$ and $B_{p q}$ are invertible. Define

$$
C_{p p}=A_{p q}^{-1} B_{p q} \quad \text { if } \quad\left|A_{p q}\right|>\left|B_{p q}\right| \quad \text { and } \quad C_{p p}=B_{p q}^{-1} A_{p q} \text { otherwise. }
$$

Clearly,

(11) $0<\left|C_{p p}\right|<1$,

(12) $C_{p p}(p)=p$,

(13) $C_{p p}$ maps a neighborhood of $p$, say $N$, onto a neighborhood of $p$. 
Since $C_{p p}$ is affine (8), (12), and (13) show that

$$
C_{p p}(H(p))=H(p)
$$

Using once more the fact that $C_{p p}$ is affine, this implies the existence of an $\alpha \in \mathbb{R}$ such that

(14) $C_{p p}(H(p)+\delta n(p))=H(p)+\alpha \delta n(p)$ for all $\delta \in \mathbb{R}$.

Next we prove

(15) $0<\alpha<1$.

Propositions (11)-(14) imply that the hyperplanes $H(p)+\delta n(p)$ and $H(p)+$ $\alpha \delta n(p)$ are in the same open half-space determined by $H(p)$ for all sufficiently small $\delta>0$. Hence $\alpha>0$. By (8) we may choose $\delta>0$ so small that the cap $C(p, \delta)$ between $H(p)$ and $H(p)+\delta n(p)$ is contained in the neighborhood $N$ of $p$ considered in (13). By (13) and (14),

(16) $C_{p p}(C(p, \delta))=C(p, \alpha \delta)$

and thus

$$
|C(p, \alpha \delta)|=\left|C_{p p}(C(p, \delta))\right|=\left|C_{p p}\right||C(p, \delta)|<|C(p, \delta)|
$$

by (11). Hence $\alpha<1$. The proof of (15) is complete.

3.3.2. We are now ready to prove that

(17) there is an $\varepsilon>0$ such that $S(p, \delta)$ is an ellipse for $0<\delta \leq \varepsilon$.

In the proof of (15) we have seen that (16) holds for all sufficiently small $\delta>0$, say for $0<\delta \leq \varepsilon$ with suitable $\varepsilon>0$. Since $S(p, \delta)$ and $S(p, \alpha \delta)$ are the boundaries of $C(p, \delta)$ and $C(p, \alpha \delta)$, respectively, we have

(18) $C_{p p}(S(p, \delta))=S(p, \alpha \delta)$ for $0<\delta \leq \varepsilon$.

Applying (18) repeatedly and taking into account (15) and (7), it follows that $S(p, \delta)$ is affine to $S\left(p, \alpha^{k} \delta\right)$ and thus to

$$
\frac{1}{\sqrt{2 \alpha^{k} \delta}}\left(S\left(p, \alpha^{k} \delta\right)-p-\alpha^{k} \delta n(p)\right)
$$

for $k=1,2, \ldots$, and $0<\delta \leq \varepsilon$. This together with (15), (9), and (1) yields the following: let $0<\delta \leq \varepsilon$. Then $S(p, \delta)$ is affine to each convex curve in a sequence of convex curves converging (with respect to $\delta^{\mathrm{H}}$ ) to the ellipse $I(p)$. Hence $S(p, \delta)$ is an ellipse itself, concluding the proof of (17).

Now apply $A_{p x}$ to transfer the property (17) from $p$ to $x$ for any $x \in S$. Hence there is $\varepsilon_{x}>0$ such that

(19) $S(x, \delta)$ is an ellipse for $0<\delta \leq \varepsilon_{x}$ and $x \in S$. 
Combining (19), (8), and (3), we see that $S$ is an ellipsoid This proves the theorem for Case 1.

3.4. Case 2. Secondly, we assume that

(20) for each pair $p, x \in S$ all affinities $A_{p x}$ have the same value of $\left|A_{p x}\right|$.

Let $p \in S$ be chosen and define functions $\varphi, \nu: S \rightarrow \mathbb{R}$ by

(21) $\varphi(x)=\log \left|A_{p x}\right|, \nu(x)=e^{\varphi(x)}$ for $x \in S$.

$\varphi$ and $\nu$ are well defined, see (7) and (20).

3.4.1. We now make some preparations for the final part of the proof in 3.4 .2 and 3.4.3. Let $\varepsilon>0$ be chosen. For $i, j=1,2, \ldots$ and $k=-i,-i+1, \ldots, i-1$, define

(22) $S_{i j k}=\left\{u \in S: \exists A_{p u}\right.$ satisfying (i)-(iii):

(i) all components of (the matrix) $A_{p u}$ are in $[-i, i]$,

(ii) $A_{p u}(N(p, 1 / i)) \supset N(u, 2 / j)$,

(iii) $\varphi(u) \in[k \varepsilon,(k+1) \varepsilon]\}$.

The next step is to prove that

(23) each set $S_{i j k}$ is closed.

To see this let $u_{1}, u_{2}, \ldots \in S_{i j k}$ converge to $u \in S$. We have to show that $u \in S_{i j k}$. By (i) all elements of the matrices $A_{p u_{i}}$ are in the interval $[-i, i]$. Hence by considering a suitable subsequence and renumbering if necessary, we may assume that

(24) $A_{p u_{i}} \rightarrow A_{p u}$ as $l \rightarrow \infty$ (elementwise),

where $A_{p u}$ is a suitable matrix. Clearly,

(25) $A_{p u}$ satisfies (i) and (iii).

In order to show that

(26) $A_{p u}$ satisfies (ii),

let $y \in N(u, 2 / j)$. Since by assumption $u_{l} \rightarrow u$ as $l \rightarrow \infty$, the inclusion $y \in N\left(u_{l}, 2 / j\right)$ holds for all sufficiently large $l$. Now, noting that $A_{p u_{i}}$ satisfies (ii), we may choose for each such $l$ a point $x_{l} \in N(p, 1 / i)$ with

(27) $A_{p u_{i}}\left(x_{l}\right)=y$.

Then, by taking a suitable subsequence and renumbering again if necessary, we may assume that

(28) $x_{l} \rightarrow x$, say, where $x \in \operatorname{cl} N(p, 1 / i)$.

Here cl stands for closure in $S$. Combining (24), (28), and (27) it follows that $A_{p u}(x)=y$. Since $y \in N(u, 2 / j)$ was arbitrary, we have proved that

(29) $A_{p u}(\mathrm{cl} N(p, 1 / i)) \supset N(u, 2 / j)$. 
By (25) $A_{p u}$ satisfies (iii). Hence $\left|A_{p u}\right|>0$. Therefore (29) yields that a fortiori

$$
A_{p u}(N(p, 1 / i)) \supset N(u, 2 / j)
$$

showing that $A_{p u}$ satisfies (iii). This proves (26). Since $A_{p u}$ satisfies (i)-(iii) (see (25), (26)), we conclude that $u \in S_{i j k}$. The proof of (23) is complete.

Since $S$ is the union of the countable system of the sets $S_{i j k}$, each of which is closed by (23), at least one of them has nonempty interior (in) $S$; this being a consequence of (4). Denoting this set by $S_{i j k}$ and let $q$ be an interior point of $S_{i j k}$.

For each $r \in S$ there are an affinity $A_{q r}$ and positive integers

(30) $l_{r} \geq j$

and $m_{r}$ such that

(31) $N\left(q, 1 / l_{r}\right) \subset S_{i j k}$,

(32) $A_{q r}\left(N\left(q, 1 / l_{r}\right)\right) \supset N\left(r, 1 / m_{r}\right)$.

The open neighborhoods $N\left(r, 1 / m_{r}\right): r \in S$ cover the compact surface $S$. Hence there is a finite number of these neighborhoods already covering $S$, say

(33) $S \subset N\left(r_{1}, 1 / m_{r_{1}}\right) \cup \cdots \cup N\left(r_{n}, 1 / m_{r_{n}}\right)$.

3.4.2. Next it is shown that

(34) $\varphi$ (and thus $\nu$ ) is continuous.

To see this we have to prove the following:

(35) Let $x \in S$ and $\varepsilon>0$ be chosen. Then there is a neighborhood $N$ of $x$ such that

$$
|\varphi(x)-\varphi(y)| \leq 2 \varepsilon \text { for each } y \in N
$$

Using the chosen value of $\varepsilon$ in 3.4.1, propositions (22) and (30)-(33) hold. By (33) there is an index $s(\leq n)$ such that

$$
x \in N=N\left(r_{s}, 1 / m_{r_{s}}\right) .
$$

Take $y \in N$. An application of (32) then shows

(36) $x=A_{q r_{s}}(u), y=A_{q r_{s}}(v)$ for suitable $u, v \in N\left(q, 1 / l_{r_{s}}\right)$.

Clearly,

(37) $u=A_{p u}(p), v=A_{p v}(p)$. 
Combining the definition of $\varphi$ in (21), assumption (20) and propositions (36), (37), (31), and (22(iii)) we obtain the following:

$$
\begin{aligned}
|\varphi(x)-\varphi(y)| & =|\log | A_{q r_{s}} A_{p u}|-\log | A_{q r_{s}} A_{p v}|| \\
& =|\log | A_{p u}|-\log | A_{p v}||=|\varphi(u)-\varphi(v)| \leq 2 \varepsilon .
\end{aligned}
$$

This concludes the proof of (35) and thus of (34).

3.4.3. Now we are ready to show that $S$ is an ellipsoid. In the following the notation of caps involving their volume is used, compare 3.1. Further, choosing $\epsilon=1$ in 3.4.1, propositions (22) and (30)-(33) are valid.

By (34) $\nu: S \rightarrow \mathbb{R}^{+}$is continuous. Hence there is an $\alpha>0$ such that

$$
0<\nu(x) \leq \alpha \quad \text { for all } \quad x \in S .
$$

Thus we may choose by (8) and (33) a $\beta>0$ which is so small that the cap

(38) $C(x, \mu \nu(x))$ is contained in one of the neighborhoods $N\left(r_{1}, 1 / m_{r_{1}}\right), \ldots$, $N\left(r_{n}, 1 / m_{r_{n}}\right)$ for each $x \in S$ and $0<\mu \leq \beta$.

Statement (38) is used to prove the following proposition:

(39) $C(x, \mu \nu(x))=A_{p x}(C(p, \mu))$ with suitable $A_{p x}$ for each $x \in S$ and $0<\mu \leq$ $\beta$.

Let $x \in S$ and $0<\mu \leq \beta$ be chosen. Then by (38) there is an index $s(\leq n)$ such that

(40) $C(x, \mu \nu(x)) \subset N\left(r_{s}, 1 / m_{r_{s}}\right)$.

Hence (7), (32), and (31) imply that

(41) $A_{q r_{s}}^{-1}(C(x, \mu \nu(x)))$ is a cap with center $u=A_{q r_{s}}^{-1}(x)$ in $N\left(q, 1 / l_{r_{s}}\right) \subset S_{i j k}$. The inclusion $u \in N\left(q, 1 / l_{r_{s}}\right)$ together with (30) and (22(ii)) yields the following:

$$
N\left(q, 1 / l_{r_{\mathrm{s}}}\right) \subset N\left(u, 2 / l_{r_{s}}\right) \subset N(u, 2 / j) \subset A_{p u}(N(p, 1 / i)) .
$$

Hence we obtain from (7) and (41) that

(42) $A_{p u}^{-1} A_{q r_{s}}^{-1}(C(x, \mu \nu(x)))$ is a cap with center $p=A_{p u}^{-1} A_{q r_{s}}^{-1}(x)$ in $N(p, 1 / i)$.

The affine transformation

$$
A_{p x}=A_{q r_{s}} A_{p u}
$$

maps $p$ onto $x$ and a neighborhood of $p$ onto a neighborhood of $x$ (since $A_{p u}, A_{q r_{s}}$ have the corresponding property). Clearly, we may rewrite (42) in the following form:

$$
A_{p x}^{-1}(C(x, \mu \nu(x))) \text { is a cap with center } p \text { in } N(p, 1 / i) .
$$


Using (20), the definition of $\nu$, and our present notation for caps, we see that its volume is

$$
\left|A_{p x}^{-1}(C(x, \mu \nu(x)))\right|=\left|A_{p x}\right|^{-1} \mu \nu(x)=\nu(x)^{-1} \mu \nu(x)=\mu .
$$

Hence

$$
A_{p x}^{-1}(C(x, \mu \nu(x)))=C(p, \mu)
$$

concluding the proof of (39).

For fixed $\mu, 0<\mu \leq \beta$, the caps

$$
C(x, \mu \nu(x)): x \in S
$$

are all affine-equivalent, see (39). Hence their boundary curves $S(x, \mu \nu(x))$ also are all affine-equivalent. It is not too difficult to show that

$$
S(x, \mu \nu(x)): x \in S
$$

depends continuously on $x$ (with respect to $\delta^{\mathbf{H}}$ ). The inverse image of the normalmapping

$$
x \rightarrow n(s): S \rightarrow S^{d-1}
$$

is continuous by (8). Hence the convex curves

$$
S\left(n^{-1}(u), \mu \nu\left(n^{-1}(u)\right)\right): u \in S^{d-1}
$$

are all affine-equivalent and depend continuously on $u$. Translating each of these curves such that its minimum circumscribed ellipse touches $S^{d-1}$ at $u$ we obtain a continuous field of affine-equivalent planar convex curves tangent to $S^{d-1}$. Applying (6) it follows that, in particular,

$$
S(x, \mu \nu(x)) \text { is centrally symmetric for each } x \in S \text { and } 0<\mu \leq \beta \text {. }
$$

Since $\nu(x)>0$, this implies that we may choose $\varepsilon_{x}>0$ such that

$$
S(x, \delta) \text { is centrally symmetric for } 0<\delta \leq \varepsilon_{x} \text { and } x \in S .
$$

Burton's result (3) now shows that $S$ is an ellipsoid, concluding the proof of the theorem for Case 2.

The proof is complete.

\section{Final Remark}

In the second part of this article the solution will be given for $d=2$ and for general $d$ under the additional assumption that the affinities are volume-preserving. 


\section{Acknowledgments}

The idea for the above result was taken from [3]. I am obliged to Professor Berger for showing it to me prior to publication. Many thanks go to Professor Chalk for his valuable comments and to Professor Stetter for directing my attention to references [11] and [21].

\section{References}

1. Alexandrov, A. D.: Almost everywhere existence of the second differential of a convex function and some properties of convex surfaces connected with it, Uchen. Zap Leningrad. Gos. Univ. Math. Ser. 6 (1939), 3-35.

2. Auerbach, H.: Sur une propriété caracteristique de l'ellipsoide, Studia Math. 9 (1940), 17-22.

3. Berger, M.: Seules les quadriques admettent des caustiques, Bull. Soc. Math. France, to appear.

4. Blaschke, W.: Über affine Geometrie XII: Von den Eiflächen, Ber. Ges. Wiss. Leipzig 70 (1918), 18-37; Gesammelte Werke, vol. 4, Thales-Verlag, Essen, 1985, pp. 157-176.

5. Brunn, H.: Über Kunven ohne Wendepunkte, Habilitationsschrift, München, 1889.

6. Burton, G. R.: On the sum of a zonotope and an ellipsoid, Comment. Math. Helv. 51 (1976), 369-387.

7. Burton, G. R.: Congruent sections of a convex body, Pacific J. Math. 81 (1979), 303-316.

8. Busemann, H.: Timelike spaces, Dissertationes Math. (Rozprawy Mat.) 53 (1967), 52.

9. Danzer, L., Laugwitz, D., Lenz, H.: Über das Löwnersche Ellipsoid und sein Analogon unter den einem Eikörper einbeschriebenen Ellipsoiden, Arch. Math. 8 (1957), 214-219.

10. Freudenthal, H.: Neuere Fassung des Riemann-Helmholtz $\sim$ Lieschen Raumproblems, Math. $Z$. 63 (1956), 374-405.

11. Golub, G. H., Van Loan, Ch. F.: Matrix Computations, Johns Hopkins University Press, Baltimore, MD, 1989.

12. Gruber, P. M.: Über kennzeichnende Eigenschaften von euklidischen Räumen und Ellipsoiden, I, J. Reine Angew. Math. 265 (1974), 61-83.

13. Gruber, P. M., Höbinger, J.: Kennzeichnungen von Ellipsoiden mit Anwendungen, in: Jahrb. Überblicke Math. 1976, Bibliographisches Institut, Mannheim, 1976, pp. 9-29.

14. Helmholtz, H. von: Über die Thatsachen, die der Geometrie zum Grunde liegen, Nachr. Ges. Wiss. Göttingen 1868, 193-221; Wissenschaftliche Abhandlungen, vol. 2, J. A. Barth, Leipzig, 1883, pp. 618-639.

15. Laugwitz, D.: Differentialgeometrie in Vektorräumen, Vieweg, Braunschweig, 1965.

16. Lie, S.: Über die Grundlagen der Geometrie, Ber. Ges. Wiss. Lepizig 42 (1890), 284-321, 355-418; Gesammelte Abhandlungen, vol. 2, Teubner, Leipzig, Aschehoug, Oslo, 1937, pp. 380-468.

17. Mani, P.: Fields of planar bodies tangent to spheres, Monatsh. Math. 74 (1970), 145-149.

18. Petty, C.: Ellipsoids, in: Convexity and Its Applications, Birkhäuser, Basel, 1983, pp. 264-276.

19. Schneider, R.: Boundary structure and curvature of convex bodies, in: Contributions to Geometry, Birkhäuser, Basel, 1979, pp. 13-59.

20. Schneider, R.: Convex Bodies: The Brunn-Minkowski Theory, Cambridge University Press, Cambridge, 1993.

21. Stewart, G. W.: Error and perturbation bounds for subspaces associated with certain eigenvalue problems, SIAM Rev. 15 (1973), 727-764.

22. Süss, W.: Kennzeichnende Eigenschaften der Kugel als Folgerungen eines Brouwerschen Fixpunktsatzes, Comment. Math. Helv. 20 (1947), 61-64.

Received March 10, 1994, and in revised form September 20, 1994. 